\title{
State-Dependent Accessibility of the P-S6 Linker of Pacemaker (HCN) Channels Supports a Dynamic Pore-to-Gate Coupling Model
}

\author{
Chung Wah Siu • Ezana M. Azene $\cdot$ Ka Wing Au • \\ Chu Pak Lau $\cdot$ Hung Fat Tse $\cdot$ Ronald A. Li
}

Received: 3 February 2009/Accepted: 11 June 2009/Published online: 17 July 2009

(c) The Author(s) 2009. This article is published with open access at Springerlink.com

\begin{abstract}
The hyperpolarization-activated cyclic nucleotide-modulated channel gene family (HCN1-4) encodes the membrane depolarizing current that underlies pacemaking. Although the topology of $\mathrm{HCN}$ resembles $\mathrm{K}_{\mathrm{v}}$ channels, much less is known about their structure-function correlation. Previously, we identified several pore residues in the S5-P linker and P-loop that are externally accessible and/or influence HCN gating, and proposed an evolutionarily conserved pore-to-gate mechanism. Here we sought dynamic evidence by assessing the functional consequences of Cys-scanning substitutions in the unexplored
\end{abstract}

C. W. Siu · R. A. Li

Stem Cell Program, University of California, Davis, CA, USA

C. W. Siu $\cdot$ R. A. Li

Department of Cell Biology and Human Anatomy, University of California, Davis, CA, USA

\section{E. M. Azene}

School of Medicine, Johns Hopkins University of School, Baltimore, MD 21205, USA

C. W. Siu - K. W. Au - C. P. Lau - H. F. Tse - R. A. Li Department of Medicine, Queen Mary Hospital, University of Hong Kong, Hong Kong, Hong Kong

R. A. Li

Institute of Pediatric Regenerative Medicine, Shriners Hospital for Children of North America, Sacramento, CA 95817, USA

C. W. Siu - K. W. Au - C. P. Lau - H. F. Tse - R. A. Li Stem Cell \& Regenerative Medicine Programme and Heart, Brain, Hormone and Healthy Aging Research Center, University of Hong Kong, Hong Kong, Hong Kong

\section{R. A. Li ( $\square)$}

Shriners Hospital, University of California, Room 650, 2425

Stockton Boulevard, Sacramento, CA 95817, USA

e-mail: ronaldli@ucdavis.edu
P-S6 linker (residues 352-359), the HCN1-R background (that is, resistant to sulfhydryl-reactive agents). None of A352C, Q353C, A354C, P355C, V356C, S357C, M358C, or S359C produced functional currents; the loss-of-function of Q353C, A354C, S357C, and M358C could be rescued by the reducing agent dithiothreitol. Q353C, A354C, and S357C, but not M358C and HCN1-R, were sensitive to $\mathrm{Cd}^{2+}$ blockade $\left(\mathrm{IC}_{50}=3-12 \mu \mathrm{M}\right.$ vs. $\left.>1 \mathrm{mM}\right)$. External application of the positively charged covalent sulfhydryl modifier MTSET irreversibly reduced $I_{-140 \mathrm{mV}}$ of Q353C and $\mathrm{A} 354 \mathrm{C}$ to $27.9 \pm 3.4 \%$ and $58.2 \pm 13.1 \%$ of the control, respectively, and caused significant steady-state activation shifts $\left(\Delta V_{1 / 2}=-21.1 \pm 1.6\right.$ for $\mathrm{Q} 353 \mathrm{C}$ and $-10.0 \pm 2.9 \mathrm{mV}$ for $\mathrm{A} 354 \mathrm{C})$. Interestingly, MTSET reactivity was also state dependent. MTSET, however, affected neither S357C nor M358C, indicating site specificity. Collectively, we have identified novel P-S6 residues whose extracellular accessibility was sterically and state dependent and have provided the first functional evidence consistent with a dynamic HCN pore-to-gate model.

Keywords Outer pore $\cdot$ Pacemaker channels $\cdot \mathrm{HCN}$

The hyperpolarization-activated cyclic nucleotide-modulated channel gene family (HCN1-4) encodes the membrane depolarizing current that underlies pacemaking (Biel et al. 2002; Brown et al. 1977; Bucossi et al. 1997; Chen et al. 2001; Creighton 1992; DiFrancesco 1981a, b, 1993, 2006; Lieu et al. 2008; Ludwig et al. 1998; Siu et al. 2006). Functionally, HCN channels nonselectively permeate $\mathrm{Na}^{+}$ and $\mathrm{K}^{+}$(with a ratio of 1:4 vs. $\leq 1: 100 \mathrm{~K}^{+}$channels) and are activated upon hyperpolarization rather than depolarization of classical voltage-gated $\mathrm{K}^{+}\left(\mathrm{K}_{\mathrm{v}}\right)$ channels (Azene et al. 2003; Prole and Yellen 2006; Siu et al. 2006). Despite 
these differences, $\mathrm{HCN}$ and $\mathrm{K}_{\mathrm{v}}$ channels have highly homologous primary amino acid sequences (Gauss et al. 1998; Giorgetti et al. 2005; Kaupp and Seifert 2001). Structurally, both are tetramers of monomeric subunits consisting of six membrane-spanning segments (S1-S6) (Gauss et al. 1998; Ludwig et al. 1998; Santoro et al. 1998; Xue et al. 2002). The region between S5 and S6, which inserts back into the membrane to form part of the ionconducting pore, can be further subdivided into three regions (Fig. 1): while the S5-P and P-S6 linkers form the extrapore mouth, the descending and ascending limbs of the P-loop, where the permeation determinant GYG motif is located, constitute the deeper region of the extracellular pore. The cytoplasmic side of the pore is believed to consist of the S6 segments (Prole and Yellen 2006).

Although the topology of $\mathrm{HCN}$ resembles $\mathrm{K}_{\mathrm{v}}$ channels, much less is known about their structure-function correlation. Previously, we identified several pore residues in the S5-P linker (C318 HCN1 numbering), the descending (H334, F339) and ascending (A352, A354) P-loop limbs that are externally accessible and/or influence $\mathrm{HCN}$ gating (Au et al. 2008; Azene et al. 2005; Xue and Li 2002). Based on these results, we proposed an evolutionarily conserved pore-to-gate mechanism (Au et al. 2008; Azene et al. 2005). However, the functional role of the P-S6 linker, putatively defined as residues 352-359 here, and its accessibility have

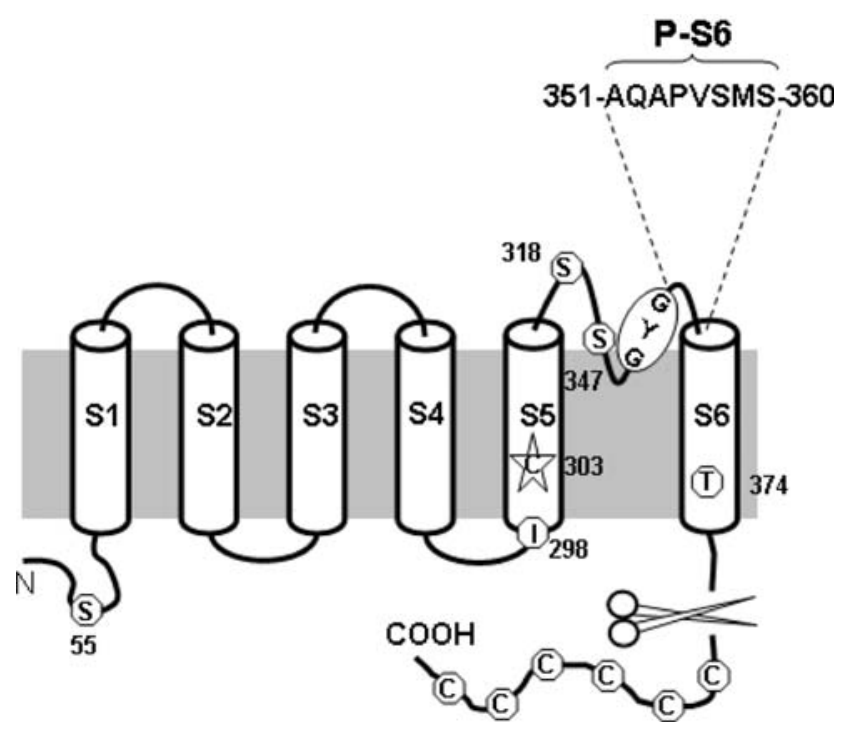

Fig. 1 Schematic representation of HCN1-R. The six transmembrane segments of a monomeric subunit of the HCN1-R are shown. Six endogenous cysteine residues are removed together with the truncation of the $-\mathrm{COOH}$ terminus. Five of the six remaining cysteine residues are replaced by a serine, threonine, or isoleucine substitution. C303 in the S5 segment is left in the final HCN1-R construct because of lack of functional current upon mutations. Their approximate locations, as well as the GYG signature motif, are highlighted as shown. The eight amino acid residues in the P-S6 motif of the final HCN1-R construct are individually replaced by cysteine and named accordingly not been explored. To obtain further insights into the footprint of the pore, here we investigated the functional consequences of systematically introducing single Cysscanning substitutions into the P-S6 linker in the background of HCN1-R channels (Bell et al. 2004), in which all endogenous cysteines except $\mathrm{C} 303$ have been removed or replaced to render the wild-type (WT) resistant to sulfhydryl-reactive agents such as Class IIB divalent $\mathrm{Cd}^{2+}$ and covalent methanethiosulfonate (MTS) modifying compounds (Karlin and Akabas 1998). Using this approach, we successfully identified several novel P-S6 linker residues whose extracellular accessibility was sterically and state dependent. These results provide the first functional evidence that the $\mathrm{HCN}$ pore undergoes dynamic rearrangements consistent with a pore-to-gate coupling mechanism.

\section{Materials and Methods}

\section{Molecular Biology}

Murine $\mathrm{HCN} 1$ and an $\mathrm{MTS} / \mathrm{Cd}^{2+}$-resistant $\mathrm{HCN} 1$ construct, HCN1-R (Bell et al. 2004), provided by Dr. Steven Siegelbaum, were subcloned into the pGHEM-HE vector (Santoro et al. 1998). The construction of HCN1-R has been described (Bell et al. 2004). In brief, 11 of 12 endogenous cysteine residues of WT HCN1 were removed or substituted: 6 cysteines were removed by truncating the $\mathrm{COOH}$ terminus and 4 of the 6 remaining cysteines were conservatively replaced by serine or threonine (i.e., C55S, C318S, C347S, and C374T), with C298 substituted by isoleucine, according to the homologous residue in cyclic nucleotide-gated (CNG) channels. As we and others have reported, substitution of C303 with any of the other 19 amino acids did not produce functional channels (Azene et al. 2003; Bell et al. 2004). Therefore, C303 was not replaced in HCN1-R. All cysteine substitutions investigated in this study were generated in the HCN1-R background using the Stratagene QuickChange site-directed mutagenesis kit (Akabas et al. 1992; Benitah et al. 1996; Karlin and Akabas 1998; Tsushima et al. 1997). The desired mutations were confirmed by DNA sequencing. cRNA was transcribed from NheI-linearized DNA using the Ambion MEGAscript transcription kit.

\section{Oocyte Preparation and Heterologous Expression}

All constructs were heterologously expressed and studied in Xenopus oocytes (Xue and Li 2002; Xue et al. 2002). Stage IV-VI oocytes were surgically removed from female Xenopus laevis anesthetized by immersion in $0.3 \%$ 3aminobenzoic acid ethyl ester, followed by digestion with $1 \mathrm{mg} / \mathrm{ml}$ collagenase (type IA) in OR-2 solution containing 
$88 \mathrm{mM} \mathrm{NaCl}, 2 \mathrm{mM} \mathrm{KCl}, 1 \mathrm{mM} \mathrm{MgCl}_{2}$, and $5 \mathrm{mM}$ HEPES (adjusted to $\mathrm{pH} 7.6$ with $\mathrm{NaOH}$ ) for $30-60 \mathrm{~min}$ After defolliculation by incubation in OR-2 solution with $10 \%$ fetal bovine serum for $15-30 \mathrm{~min}$, isolated oocytes were injected with cRNA (50 ng/oocyte) and incubated in ND96 solution containing $96 \mathrm{mM} \mathrm{NaCl}, 2 \mathrm{mM} \mathrm{KCl}$, $1.8 \mathrm{mM} \mathrm{CaCl}_{2}, 1 \mathrm{mM} \mathrm{MgCl}_{2}$, and $5 \mathrm{mM}$ HEPES (adjusted to $\mathrm{pH} 7.6$ with $\mathrm{NaOH}$ ) supplemented with $50 \mu \mathrm{g} / \mathrm{ml}$ gentamycin, $5 \mathrm{mM}$ pyruvate, and $0.5 \mathrm{mM}$ theophylline for 1-2 days before experiment.

Preparation of Oocyte Membrane Fractions and Western Blotting

As we previously described, injected and control (uninjected) oocytes were homogenized in ice-cold PBS containing an EDTA-free protease inhibitor cocktail (1 tablet/ $10 \mathrm{ml}$; Roche Applied Science) and centrifuged for $10 \mathrm{~min}$ at $1000 \mathrm{~g}$ to remove pigment and nuclear and mitochondrial materials. The supernatant was fractioned into cytosolic and membrane fractions by ultracentrifugation at $120,000 \mathrm{~g}$ for $1 \mathrm{~h}$ at $4^{\circ} \mathrm{C}$. The membrane fraction was resuspended in $1 \times$ LDS sample buffer and electrophoresed on a $10 \%$ polyacrylamide-SDS gel. Membrane proteins were then transferred onto a nitrocellulose membrane, followed by blockade with $5 \%$ nonfat dried milk for $1 \mathrm{~h}$. Blocked membranes were washed in Tris-buffered saline-Tween (TBST) and incubated with a purified rabbit polycolonal anti-HCN1 antibody (Alomone Labs, Jerusalem, Israel) at a 1:200 dilution for $4^{\circ} \mathrm{C}$ overnight, followed by a wash in TBST and an incubation with horseradish peroxidaseconjugated donkey anti-rabbit immunoglobulin (Chemicon, Temecula, CA) diluted to $1: 10,000$ in $5 \%$ nonfat dried milk for $1 \mathrm{~h}$ at room temperature. Membranes were washed again three times in TBST, and bound secondary antibodies were detected with an ECL detection kit (Chemicon) by autoradiography.

\section{Electrophysiology}

Two-electrode voltage-clamp recordings were performed at room temperature using a Warner OC-725B amplifier 12 days after cRNA injection. Measuring electrodes (TW120-6; World Precision Instruments Inc., Sarasota, FL) were pulled using a Sutter micropipette puller P-97 with final tip resistances of 2-4 M $\Omega$ when filled with $3 \mathrm{mM}$ $\mathrm{KCl}$. The recording bath solution contained $96 \mathrm{mM} \mathrm{KCl}$, $2 \mathrm{mM} \mathrm{NaCl}, 2 \mathrm{mM} \mathrm{MgCl}$, and $10 \mathrm{mM}$ HEPES (adjusted to $\mathrm{pH} 7.5$ with $\mathrm{NaOH}$ ).

For the steady-state current-voltage $(I-V)$ protocol, whole-cell currents were measured at the end of a 3-s pulse from -140 to $0 \mathrm{mV}$ from a holding potential of $-30 \mathrm{mV}$ against the test potentials. The voltage dependence of $\mathrm{HCN}$ channel activation was assessed by plotting tail currents measured immediately after a pulse to $-140 \mathrm{mV}$ as a function of the preceding 3-s test pulse normalized to the tail current at $-140 \mathrm{mV}$. Data were fitted to the Boltzman function using the Marquardt-Levenberg algorithm in a nonlinear least-squares procedure:

$m_{\infty}=1 /\left(1+\exp \left(\left(V_{\mathrm{t}}-V 1 / 2\right) / k\right)\right)$

where $V_{t}$ is the test-voltage, $V_{1 / 2}$ is the midpoint, and $k=R T / z F$ is the slope factor of steady-state activation $\left(m_{\infty}\right)$, and $R, T, z$, and $F$ have their usual meanings.

For tail $I-V$ relationships, currents were recorded immediately after stepping to a family of test voltages ranging from -100 to $+40 \mathrm{mV}$ preceded by a 3-s prepulse to either -140 or $-20 \mathrm{mV}$. The difference in tail currents resulting from the two prepulse potentials was plotted against the test potentials and fitted with linear regression to obtain the reversal potential $\left(E_{\text {rev }}\right)$.

To test for $\mathrm{Cd}^{2+}$ sensitivity, each oocyte expressing an HCN1r pore cysteine mutant was first stimulated by a 2-s voltage pulse from a holding voltage of $0 \mathrm{mV}$ to a test voltage of $-140 \mathrm{mV}$. The steady-state current at $-140 \mathrm{mV}$ was used as the initial current magnitude against which currents after $\mathrm{Cd}^{2+}$ exposure were compared. Immediately after the initial test pulse, cells were exposed to varying concentrations of $\mathrm{CdCl}_{2}(3 \mu \mathrm{M}$ to $1 \mathrm{mM})$. Voltage pulses (from 0 to $-140 \mathrm{mV}, 2$-s duration) were then applied every minute until current block reached an equilibrium level. The fractional block by $\mathrm{Cd}^{2+}$ was plotted against the concentration to generate a binding curve. Data were fitted to the Hill equation using the Marquardt-Levenberg algorithm in a nonlinear least-squares procedure:

$F_{\text {blocked }}=I_{\min }+\frac{I_{\max }-I_{\min }}{1+\left(\frac{\left[\mathrm{CdCl}_{2}\right]}{\mathrm{IC}_{50}}\right)^{h}}$

where $F_{\text {blocked }}$ is the fractional current block, $I_{\min }$ is the minimum fractional current block, $I_{\max }$ is the maximum fractional current block, $\mathrm{IC}_{50}$ is the (Benitah et al.) that led to $50 \%$ fractional block, and $h$ is the Hill coefficient.

2-Trimethylammoniumethylmethane thiosulfonate (MT SET; Toronto Research Chemicals, Toronto, ON, Canada) powder was dissolved in deionized water at $0.2 \mathrm{M}$ shortly before experiments. The stock solution was stored on ice and used within $1 \mathrm{~h}$. The MTSET shock solution was diluted with the bath solution to $2 \mathrm{mM}$ and applied extracellularly to the oocytes after control data were obtained. The time course of MTSET modification was fit with the following single-exponential equation:

$F=(1-S) \times \exp \left(-t / \tau_{\text {MTSET }}\right)+S$

where $F$ is the fraction of remaining current measured at $-140 \mathrm{mV}$ in the presence of MTSET, $t$ is the cumulative 
exposure time, $\tau_{\text {MTSET }}$ is the time constant for MTSET modification, and $S$ is the steady-state plateau (Xue and $\mathrm{Li}$ 2002).

All data reported are mean \pm SE. Statistical significance was determined from all individual data points and fitting parameters using one-way ANOVA and the Tukey HSD post hoc test at the 5\% level.

\section{Results}

As our first step, we characterized and compared the baseline properties of WT and HCN1-R channels. Figure $2 \mathrm{a}$ shows representative current tracings of WT and HCN1-R channels recorded using the electrophysiological protocol given in the inset. Consistent with previous studies (Au et al. 2008; Bell et al. 2004), oocytes injected with HCN1-R cRNA robustly expressed hyperpolarizationactivated currents comparable to those of WT $\left(I_{-140 \mathrm{mV}}=\right.$ $-12.3 \pm 2.5$ and $-15.6 \pm 3.3 \mu \mathrm{A}$, respectively; $p>$ $0.05)$. The corresponding current-voltage relationships are given in Fig. 2b. Figure 2c shows their steady-state activation curves. In comparison to WT, HCN1-R channels displayed a modest but insignificant hyperpolarizing shift of their activation midpoint $\left(V_{1 / 2}=-80.3 \pm 2.5 \mathrm{mV}\right.$ $[n=6]$ vs. $86.4 \pm 1.7 \mathrm{mV}[n=5]$ of WT; $p>0.05)$. The slope factor $k$ was also not different $(17.2 \pm 0.6$ and $16.2 \pm 0.5$, respectively; $p>0.05$ ). These activation gating parameters are summarized in Table 1.

\section{Effect of Dithiothreitol (DTT) on P-S6 Cys-Substituted HCN1 Channels}

Unlike WT and HCN1-R, none of the eight Cys-substituted constructs A352C, Q353C, A354C, P355C, V356C, S357C, M358C, and S359C produced functional currents (Fig. 3a). Since HCN channels are tetrameric, and only the cRNA of a single Cys-substituted construct was injected, each expressed channel protein carried four copies of the introduced cysteine. If these introduced sulfhydryls in the pore are in close enough geometric proximity, it is possible that they cross-link and thereby restrict the pore for permeation. To test this possibility, we incubated injected oocytes with the reducing agent DTT $(2.5 \mathrm{mM})$. Interestingly, the loss of function of Q353C, A354C, S357C, and M358C could be rescued by DTT, as evident by the presence of hyperpolarization-activated currents (Fig. 3b). By contrast, A352C, P355C, V356C, and S359C failed to produce measurable currents even after DTT treatment. As we previously reported (Au et al. 2008), DTT had no detectable effect on the steady-state $I-V$ relationship and activation gating of WT and HCN1-R channels (data not shown). The steady-state activation curves of DTT-treated
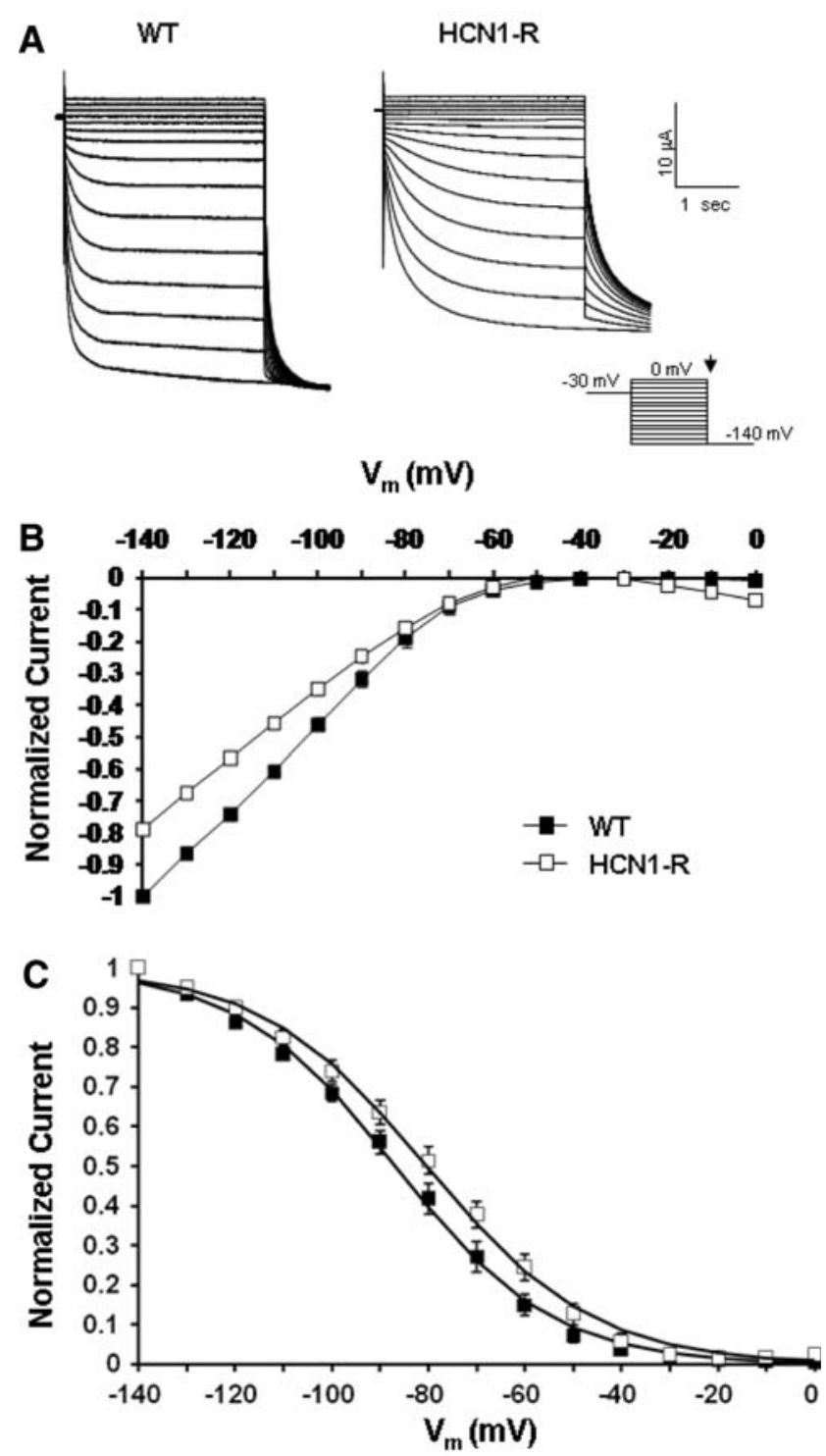

Fig. 2 a Representative current tracings of WT HCN1 and HCN1-R. Currents were elicited by stepping to a family of 3-s electrical pulses ranging from 0 to $-140 \mathrm{mV}$ in $10-\mathrm{mV}$ increments from a holding potential of $-30 \mathrm{mV}$. b Steady-state current-voltage $(I-V)$ relationships (at 3 s). c Steady-state activation curves

HCN1-R, Q353C, A354C, S357C, and M358C are given in Fig. 3c. While the steady-state activation gating properties of Q353C and A354C were not statistically different from HCN1-R, those of S357C and M358C were depolarized $(p<0.05)$. All gating parameters are summarized in Table 1.

Effect of $\mathrm{Cd}^{2+}$ on Cys-Substituted HCN1 Constructs

Given the high-affinity binding of class II divalents to free sulfhyfryl, we next examined the sensitivity of HCN1R, Q353C, A354C, S357C, and M358C channels for current blockade by $\mathrm{Cd}^{2+}$ (Fig. 4). Representative current tracings 
Table 1 Summary of steady-state activation properties of WT, HCN1-R, and various P-S6 mutants

\begin{tabular}{lllr}
\hline Channel & $V_{1 / 2}(\mathrm{mV})$ & $K$ & $N$ \\
\hline WT HCN1 & $-86.4 \pm 1.7$ & $16.2 \pm 0.5$ & 5 \\
HCN1-R & $-80.3 \pm 2.5$ & $17.2 \pm 0.6$ & 6 \\
Q353C $^{\mathrm{a}}$ & $-91.3 \pm 1.2$ & $14.4 \pm 0.5$ & 12 \\
A354C & $-87.4 \pm 1.0$ & $18.3 \pm 0.9$ & 15 \\
S357C $^{\text {a }}$ & $-75.5 \pm 1.4^{*}$ & $16.1 \pm 0.5$ & 5 \\
M358C $^{\text {a }}$ & $-69.2 \pm 0.6^{*}$ & $11.5 \pm 0.5$ & 9 \\
\hline
\end{tabular}

${ }^{\text {a }}$ Measurable currents are only produced with $24-\mathrm{h}$ incubation with DTT

* Statistically different $(p<0.05)$ from WT HCN1 channels recorded in the absence and presence of $\mathrm{Cd}^{2+}$ are given. Consistent with previous reports, HCN1-R channels were completely insensitive to $\mathrm{Cd}^{2+}(n=3)$ (Au et al. 2008). Similarly, $\mathrm{Cd}^{2+}$ of up to $1000 \mu \mathrm{M}$ led to no current blockade of M358C channels at $-140 \mathrm{mV}(n=3)$. By contrast, $100 \mu \mathrm{M} \mathrm{Cd}^{2+}$ sufficed to reduce $\mathrm{I}_{-140 \mathrm{mV}}$ of $\mathrm{S} 357 \mathrm{C}$ to $23 \pm 5 \%(n=3)$ and even completely blocked Q353C $(n=4)$ and A354C $(n=4)$ channels. Steady-state $I-V$ relationships of $\mathrm{Q} 353 \mathrm{C}, \mathrm{A} 354 \mathrm{C}$, and $\mathrm{S} 357 \mathrm{C}$ recorded in the presence of $\mathrm{Cd}^{2+}$ (at $\mathrm{IC}_{50}$ ) did not shift the $V_{1 / 2}$ of Q353C $\left(\Delta V_{1 / 2}=-4.6 \pm 1.4 \mathrm{mV}\right), \mathrm{A} 354 \mathrm{C}(-1.2 \pm 0.8 \mathrm{mV})$, and S357C $(-0.1 \pm 0.2 \mathrm{mV})(p>0.05)$, suggesting that the observed current reductions were not secondary to changes
Fig. 3 Representative current tracings of the eight P-S6 Cyssubstituted constructs (a) before and (b) after DTT. c Steadystate activation curves of Q353C, A354C, S357C, and M358C after DTT treatment

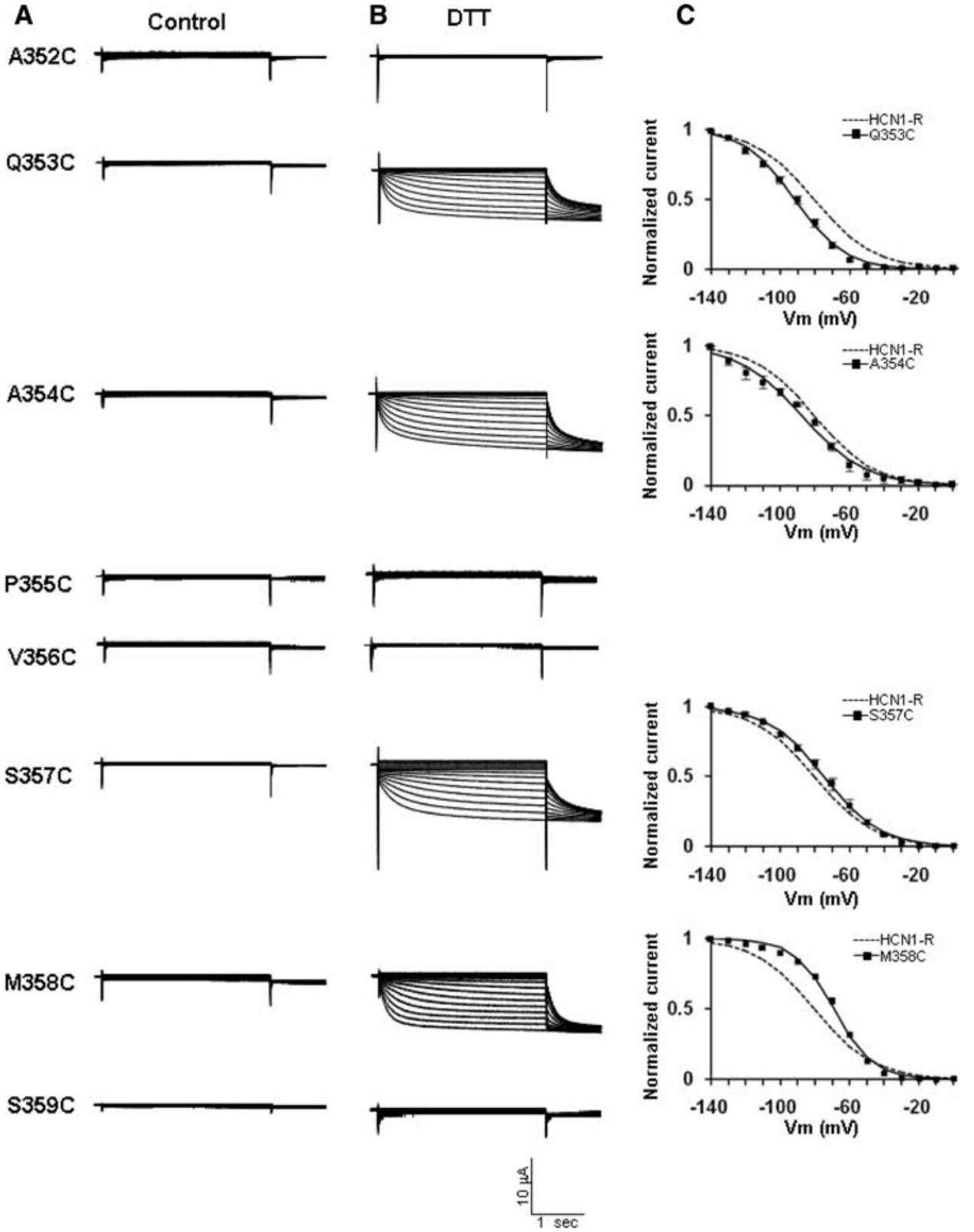



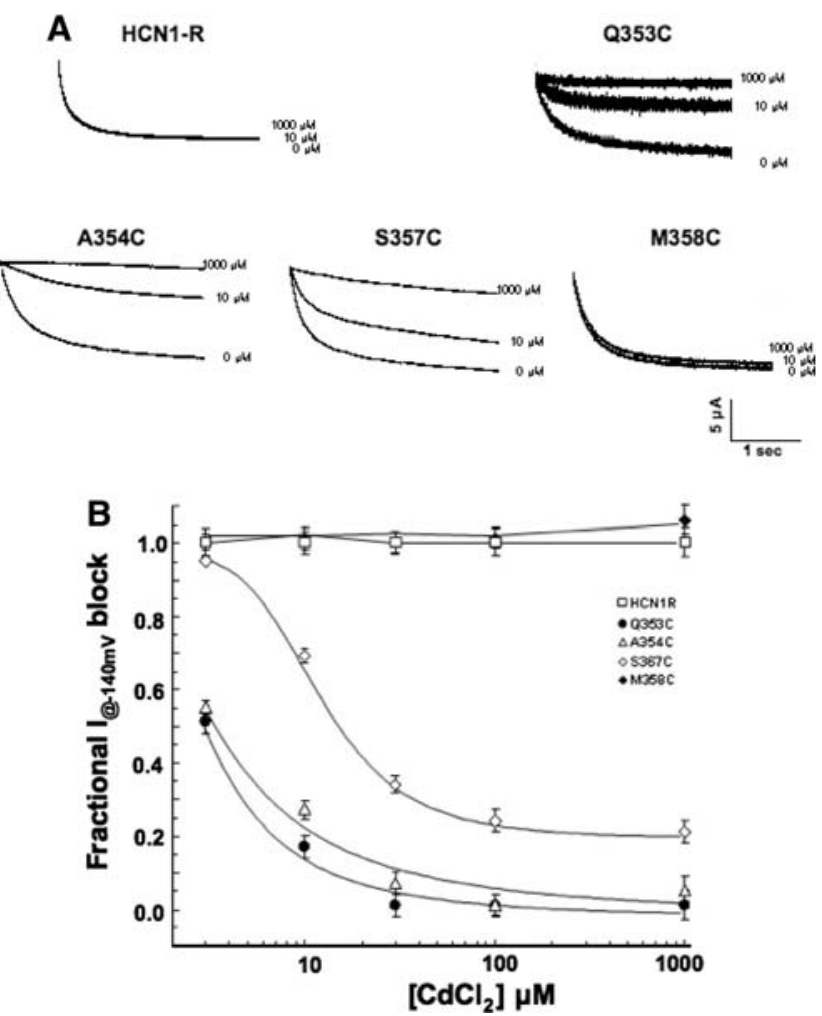

Fig. 4 Effects of $\mathrm{Cd}^{2+}$ on HCN1-R, Q353C, A354C, S357C, and M358C channels. a Representative current tracings measured at $140 \mathrm{mV}$ in the absence and presence of $\mathrm{Cd}^{2+}$ as indicated. b Doseresponse relationships

in gating properties. Figure $4 \mathrm{~b}$ shows the dose-response relationships of current inhibition by $\mathrm{Cd}^{2+}$ block for HCN1-R, Q353C, A354C, S357C, and M358C channels. From these binding curves, the $\mathrm{IC}_{50}$ values for $\mathrm{Cd}^{2+}$ block of Q353C, A354C, and S357C were $3.1 \pm 1.1(n=4)$, $3.7 \pm 1.1(n=3)$, and $12.4 \pm 1.0(n=3) \mu \mathrm{M}$, respectively (Table 2). Interestingly, a residual component for S357C was observed at high concentrations. The relationships for HCN1-R and M358C were flat across the concentration range examined.

\section{Covalent Modification of P-S6 Pore Cys-Substituted Constructs by MTSET}

To complement our $\mathrm{Cd}^{2+}$ experiments, we next examined the effect of the permanently charged, membrane-impermeant covalent sulfhydryl modifier MTSET (Figs. 5-7 and Table 3). Since the size of MTSET is known $(5.8 \times 5.8 \times 10 \AA)$, successful modification of the inserted sulfhydryl would enable us to estimate the lower limit of the size of the accessible extrapore region. Similar to the MTS-resistant HCN1-R (Au et al. 2008; Bell et al. 2004), external application of $2.5 \mathrm{mM}$ MTSET to $\mathrm{Cd}^{2+}$-resistant M358C channels did not result in any observable changes
Table 2 Summary of effects of $\mathrm{CdCl}_{2}$ on Q353C, A354C, and S357C channels

\begin{tabular}{lrlll}
\hline Channel & $\mathrm{IC}_{50}(\mu \mathrm{M})$ & $\begin{array}{l}\text { Hill } \\
\text { coefficient }\end{array}$ & \multicolumn{1}{l}{$\begin{array}{l}V_{1 / 2}(\mathrm{mV}) \text { at }\left[\mathrm{CdCl}_{50}\right] \text { of } \\
\text { [353C }\end{array}$} & $N$ \\
\hline $\mathrm{A} 354 C^{\mathrm{a}}$ & $3.1 \pm 1.1$ & 1.55 & $-89.3 \pm 1.5$ & 3 \\
$\mathrm{~S}_{557 C^{\mathrm{a}}}$ & $12.4 \pm 1.1$ & 1.15 & $-85.7 \pm 0.9$ & 4 \\
\hline
\end{tabular}

${ }^{\text {a }}$ Measurable currents are only produced with 24-h incubation with DTT

in the whole-cell currents, gating parameters, and reversal potentials (Fig. 6; $p>0.05$ ). Interestingly, $\mathrm{Cd}^{2+}$-sensitive S357C channels were also resistant to MTSET modification (Fig. 6; $p>0.05$ ). In stark contrast, MTSET application led to a significant current reduction at steady state at $-140 \mathrm{mV}$ of $\mathrm{Q} 353 \mathrm{C} \quad\left(I_{\mathrm{MTSET}} / I_{\mathrm{Control}}=27.9 \pm 3.4 \%\right.$; $n=5)$ and A354C (58.2 $\pm 13.1 \% ; n=8)$ (Figs. 5 and $7 \mathrm{a}$; $p<0.05)$.

Since current reduction induced by MTSET could result from changes in the permeation pathway, gating properties, or both, we next studied the effect of MTSET on steady-state activation to distinguish among these possibilities. Figure 7b showed that MTSET modification similarly and significantly shifted the activation curves of Q353C $\left(V_{1 / 2, \text { MTSET }}=-103.3 \pm 2.0 \mathrm{mV}\right.$ vs. $V_{1 / 2, \text { Control }}=$ $-82.2 \pm 0.9 \mathrm{mV} \quad[n=7] ; \quad p<0.01) \quad$ and $\mathrm{A} 354 \mathrm{C}$ $\left(V_{1 / 2, \mathrm{MTSET}}=-96.3 \pm 2.1 \mathrm{mV}\right.$ vs. $V_{1 / 2, \text { Control }}=-86.3$ $\pm 1.9 \mathrm{mV}[n=8] ; p<0.05)$ channels in the hyperpolarizing direction. Importantly, the steady-state activation plateau was reached at $-140 \mathrm{mV}$ for both constructs, implying that macroscopic $I_{-140 \mathrm{mV}}$ reductions observed could primarily result from gating modification. However, an effect on permeation cannot be excluded given that the open probability of $\mathrm{HCN}$ channels may not reach $100 \%$ even at saturating hyperpolarizing potentials (Flynn et al. 2007; Chen et al. 2007). In general, these phenotypes of Q353C and A354C were similar to our previously reported S5-P C318 whose MTS-induced reduction is primarily attributed to the hyperpolarizing activation shift (Xue and Li 2002). MTSET also slowed the activation and deactivation kinetics (Table 3) of Q353C and A354C but without altering their reversal potentials (Fig. 7c).

\section{State-Dependent MTSET Modification of Q353C} and A354C

The Shaker residues M448 and P450, whose analogous residues in HCN1 are Q353 and P355, respectively, have been shown to become highly accessible or even capable of cross-linking in the C-type inactivated state (Liu et al. 1996). Given the demonstrated accessibility of Q353C and the proximity of A354 to P355 (whose Cys substitution led 
Fig. 5 Representative current tracings of Q353C, A354C, S357C, and M358C before and after external application of MTSET

\section{Control}

MTSET

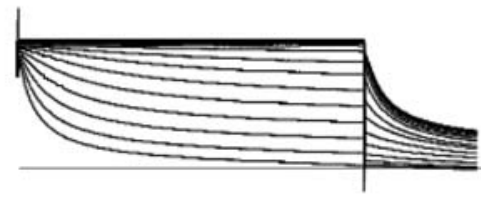

$5 \mu \mathrm{A}$

Q353C

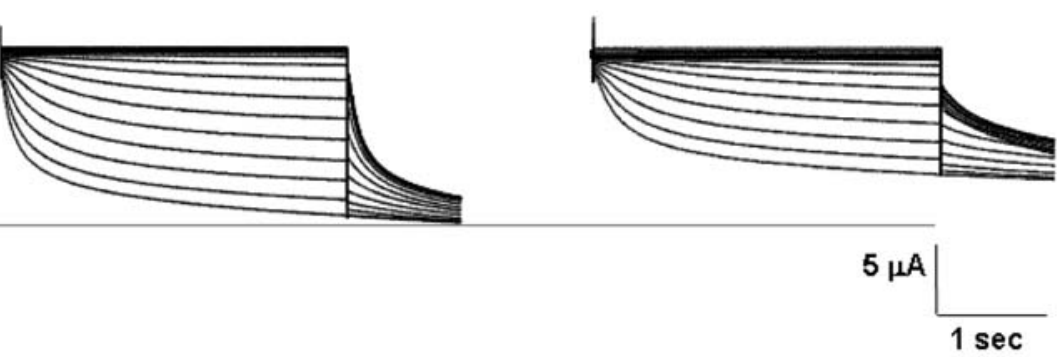

S357C
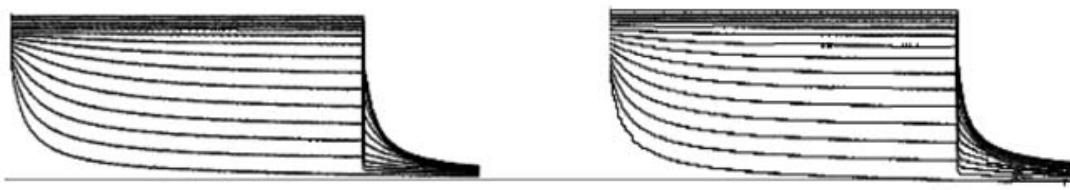

$2.5 \mu \mathrm{A}$

$1 \mathrm{sec}$

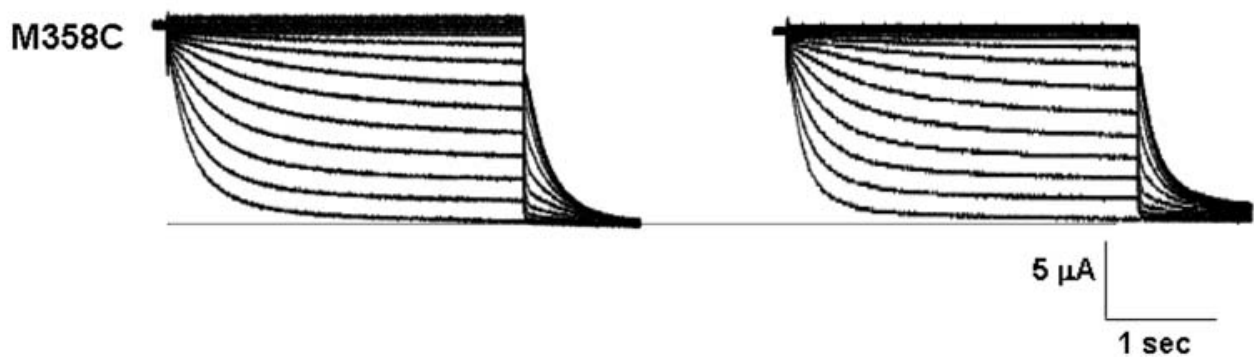

to dysfunctional channels; cf. Fig. 3), we next examined if MTSET modification of Q353C and A354C channels was state dependent. With a stimulation frequency of $0.03 \mathrm{~Hz}$, $I_{-140 \mathrm{mV}}$ of Q353C reduced progressively upon external MTSET application (Fig. 8a). The modification time constant $\left(\tau_{\text {MTSET }}\right)$ was $381.6 \pm 24.7 \mathrm{~s}(n=3)$, with steadystate block achieved $\sim 12$ min after application. However, when the stimulation frequency was slowed to $0.016 \mathrm{~Hz}$, steady-state modification required $\sim 20 \mathrm{~min}$ and $\tau_{\text {MTSET }}$ significantly increased to $892.6 \pm 82.1 \mathrm{~s} \quad(n=3$; $p<0.05$ ). Similarly, MTSET modification of A354C was also state dependent (Fig. 8b). At $0.03 \mathrm{~Hz}, \tau_{\text {MTSET }}$ was $364.1 \pm 35.7 \mathrm{~s}$ and steady-state block was completed at $\sim 8 \mathrm{~min}$. These were changed to $693.3 \pm 61.5 \mathrm{~s}$ and $\sim 20 \mathrm{~min}$, respectively, when the slower frequency of $0.016 \mathrm{~Hz}$ was applied. Interestingly, no apparent differences were observed for both Q353C and A354C channels when normalized $I_{-140 \mathrm{mV}}$ was plotted against the pulse number (rather than time) (Fig. 8c and d). These data implicate that residues $\mathrm{C} 353$ and $\mathrm{C} 354$ were reactive to MTSET only during the open state when depolarized. Taken collectively, our observations were consistent with the pore-to-gate coupling hypothesis that the P-S6 extrapore region undergoes dynamic conformational changes during gating.

A352C, P355C, V356C, and S359C channels were dysfunctional but synthesized.

To further explore the basis of the loss-of-function phenotype of A352C, P355C, V356C, and S359C channels even after DTT treatment, we performed Western blot analysis (Fig. 9). For WT and HCN1-R channels prepared under control (oxidizing) conditions and after DTT incubation, only single bands at $76 \mathrm{kDa}$, as expected for the size for the HCN1 monomer, were detected. Without DTT, additional bands at $150 \mathrm{kDa}$ that appeared to be crosslinked dimers were seen for A352C, Q353C, V356C, S357C, and S359C. However, similar dimers were not seen for A354C, P355C, and M358C channels. Interestingly, all 
Fig. 6 a Steady-state $I-V$ relationships of S357C and M358C before and after MTSET. b Steady-state activation curves. c Tail $I-V$ relationships
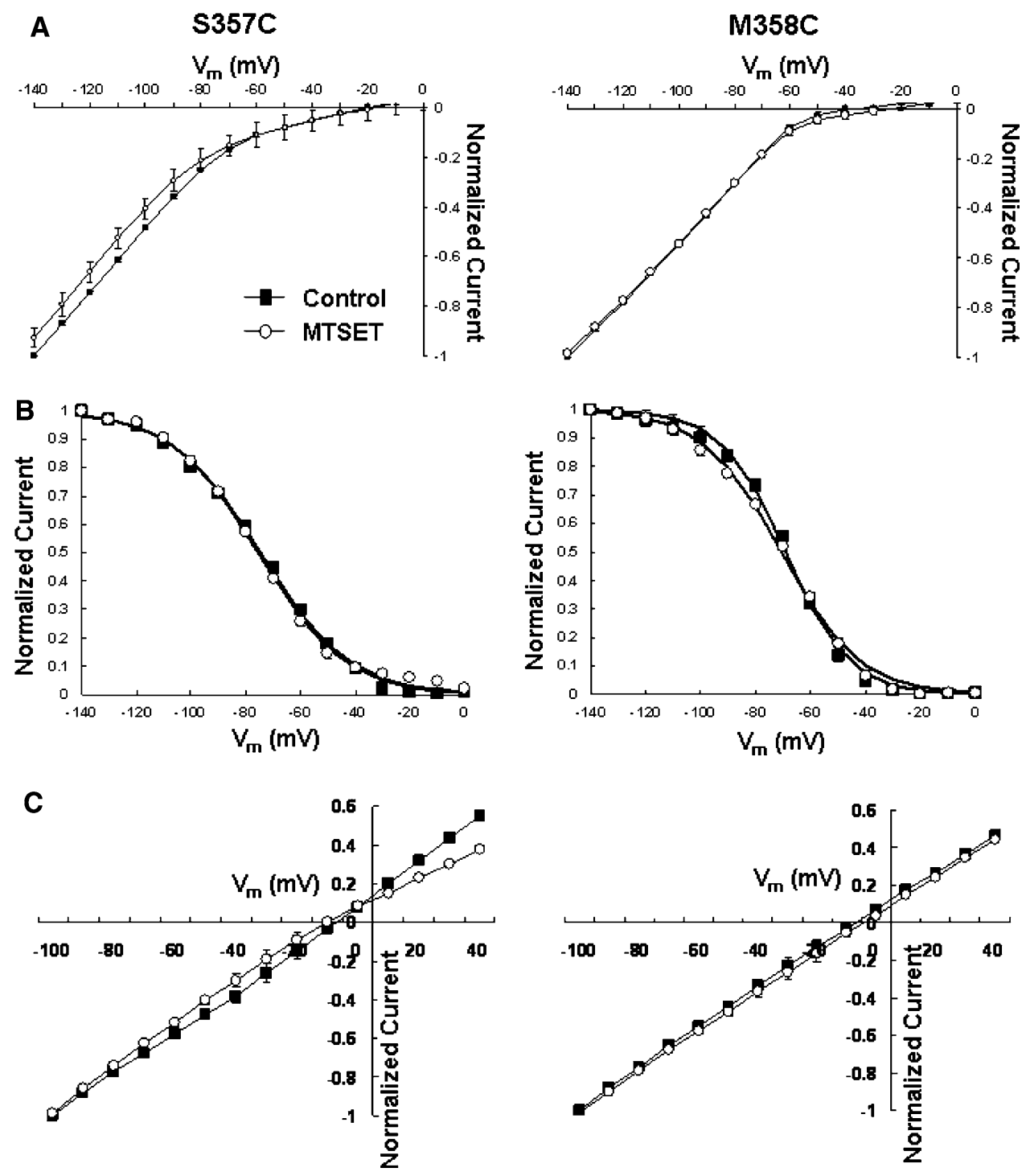

dimers disappeared after DTT treatment, and only monomers similar to those of WT and HCN1-R channels were observed. All constructs displayed bands with intensity comparable with those of WT and HCN1-R channels (with the identical amounts of cRNA injected), suggesting that protein synthesis and translational efficiency were not affected by the Cys substitutions. The same pattern was observed in three other experiments performed independently with different batches of Xenopus oocytes.

\section{Discussion}

In the present study, we employed Cys-scanning substitutions to investigate the previously unexplored P-S6 linker that is C-terminal to the GYG motif. Based on our results, we conclude that the P-S6 linker residues 352-359 are important determinants of the structure-function properties of HCN1 channels. Among these pore residues, Q353, A354, and S357 are exposed to the aqueous phase as gauged by $\mathrm{Cd}^{2+}$ and/or MTSET sensitivities. The accessibility of Q353 and A354 is state dependent, consistent with an allosteric pore-to-gate coupling model that involves dynamic rearrangements of the extrapore. Using the same approach, we previously identified the endogenous cysteine $\mathrm{C} 318$ from the S5-P linker (Xue and Li 2002) and H344 from the descending limb of the P-loop (Au et al. 2008) immediately N-terminal to GYG as extracellularly accessible residues. The collective data sets provide structural and functional insights for deducing a more comprehensive footprint of the HCN pore, as further discussed below.

The $\mathrm{Cd}^{2+}$-sensitive but MTSET-resistant S357C channels were phenotypically distinctive from Q353C and A354C in that the latter were sensitive to both. Furthermore, $\mathrm{Cd}^{2+}$ led to complete blockade of Q353C and 
Fig. 7 a Steady-state $I-V$ relationships of Q353C and A354C before and after MTSET. b Steady-state activation curves. c Tail $I-V$ relationships
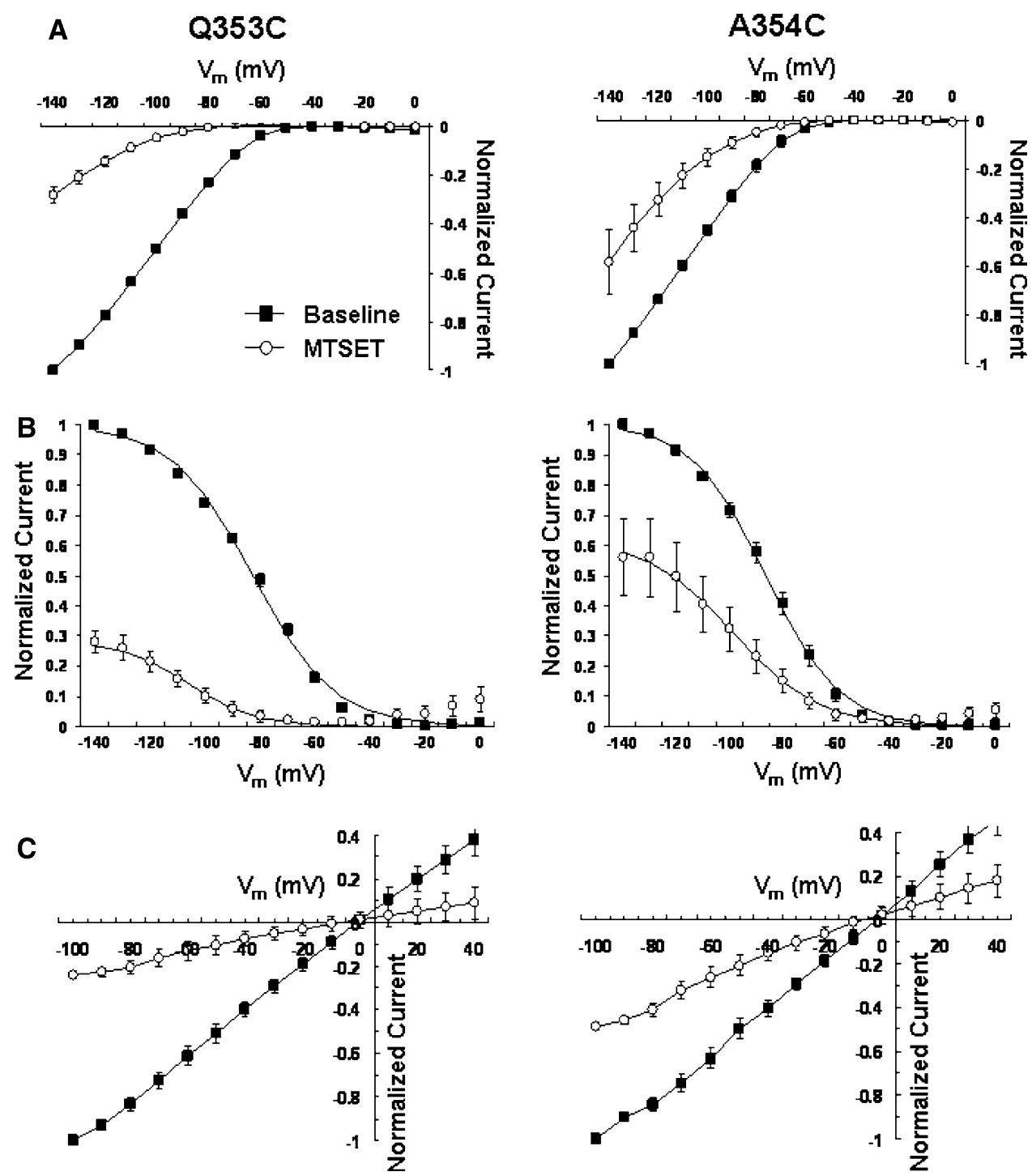

Table 3 Summary of activation and deactivation kinetics of WT and mutant HCN1 channels before and after MTSET modification

\begin{tabular}{|c|c|c|c|c|c|}
\hline Mutant & $\tau_{\text {activation }}$ & $\tau_{\text {deactivation }}$ & $\tau_{\text {activation (MTSET) }}$ & $\tau_{\text {deactivation (MTSET) }}$ & $I_{\mathrm{MTSET}} / I_{\text {Control }}$ \\
\hline HCN1R & $371 \pm 6$ & $65 \pm 10$ & $390 \pm 6$ & $64 \pm 9$ & $100.3 \pm 4.9 \%$ \\
\hline Q353C & $403 \pm 40$ & $50 \pm 3$ & $1212 \pm 228$ & $236 \pm 10$ & $27.9 \pm 3.4 \%$ \\
\hline A354C & $444 \pm 12$ & $353 \pm 23$ & $1488 \pm 57$ & $537 \pm 54$ & $58.2 \pm 13.1 \%$ \\
\hline S357C & $661 \pm 13$ & $318 \pm 17$ & $729 \pm 10$ & $341 \pm 11$ & $97.0 \pm 4.7 \%$ \\
\hline M358C & $320 \pm 6$ & $79 \pm 6$ & $322 \pm 2$ & $77 \pm 7$ & $96.0 \pm 3.3 \%$ \\
\hline
\end{tabular}

A354C but a residual component $(\sim 20 \%)$ remained for S357C even at high concentrations (cf. Fig. 4). Our findings were also consistent with previously reported cysteinescanning experiments of the sea urchin sperm (sp) HCN channels, whose F434 residue homologous to HCN1 Q353 when replaced by cysteine similarly enhanced macroscopic current blockage by extracellular $\mathrm{Cd}^{2+}$ (Roncaqlia et al. 2002). A possible explanation for these observations is that the side chain of $\mathrm{S} 357 \mathrm{C}$ is indeed exposed to the extracellular milieu but at a location that is more superficial than those of Q353 and A354 (Fig. 10). As such, $\mathrm{Cd}^{2+}$ binding to $\mathrm{C} 357$ does not lead to complete occlusion of the pore. Functionally, however, the bound $\mathrm{Cd}^{2+}$ can inhibit conduction by electrostatic repulsion of the incoming permeant ions. Also, as a result of its superficial location in the extrapore, even successful MTSET modification cannot lead to functional changes because (i) the ethyl linker that attaches the MTS moiety to $\mathrm{C} 357$ precludes direct pore 

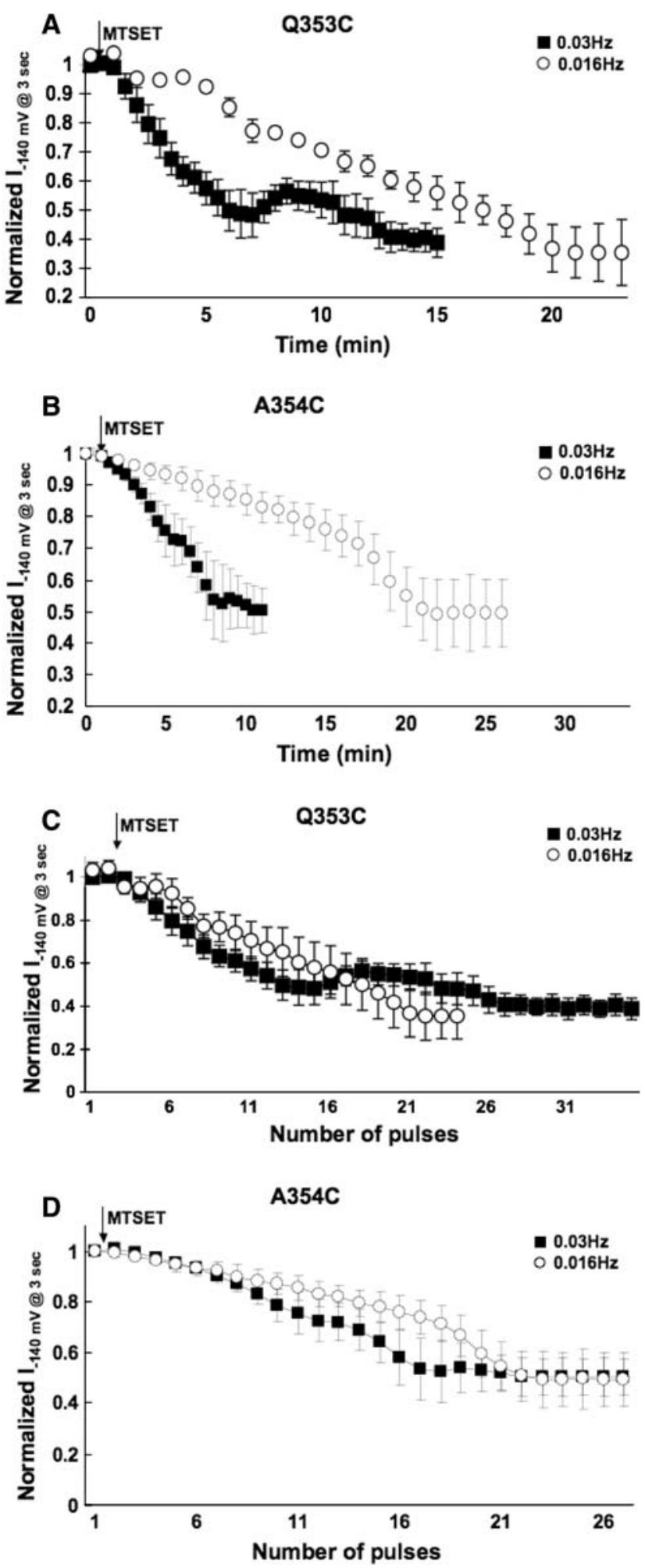

Fig. 8 Time course of the development of current inhibition at $140 \mathrm{mV}$ upon external application of $2.5 \mathrm{mM}$ MTSET with a stimulation frequency of 0.03 and $0.016 \mathrm{~Hz}$ to $\mathbf{a} \mathrm{Q} 353 \mathrm{C}$ and $\mathbf{b}$ A354C channels. c, $\mathbf{d}$ The same experimental data from A and B are plotted as normalized $I_{-140 \mathrm{mV}}$ against the pulse number (rather than time)

occlusion and (ii) residue 357 is not involved in dynamic pore rearrangements during gating, unlike Q353C, A354C, and $\mathrm{C} 318 \mathrm{C}$. By contrast, MTS modification of Q353C or A354C occludes the pore, increases the energy required for channel opening (as reflected by a hyperpolarizing shift of $V_{1 / 2}$ ), and slows the gating kinetics (due to the attachment of the bulky MTS moiety to a dynamic pore region).

Unlike Q353C, A354C, and S357C, however, M358C has a phenotype that is similar to the $\mathrm{F} 339 \mathrm{C}$ ( $\mathrm{N}$ terminal to the GYG motif in the $\mathrm{P}$ region) that we previously described ( $\mathrm{Au}$ et al. 2008). Both M358C and F339C channels displayed loss-of-function that could be rescued by DTT, but the restored currents were sensitive to neither $\mathrm{Cd}^{2+}$ nor MTSET. We cannot exclude the possibility that $\mathrm{Cd}^{2+}$ binding or MTSET modification of M358C does not lead to functional changes due to its distance from the permeation pathway (particularly given the proposed superficial location of S357). Further experiments will be needed to dissect the underlying mechanisms. The lack of measurable functional currents from A352C, P355C, V356C, and S359C channels, however, did not enable us to assess their accessibility.

Our present results were consistent with our previously proposed pore-to-gate coupling model of HCN channels (Azene et al. 2003, 2005; Xue and Li 2002) and provide further structural and functional insights. To date, we have identified that $\mathrm{C} 318$ from the S5-P linker (Xue and Li 2002), H344 of the ascending arm of the P-loop (Au et al. 2008), and Q353, A354, and S357 of the P-S6 segment (from the present study) are exposed to the aqueous phase 
Fig. 10 Schematic

representation of the $\mathrm{HCN}$ pore summarizing our present results and those of our two previously published studies. Gray circles, cysteinyl side chain; open circles, permeant ion; filled circles, endogenous amino acid
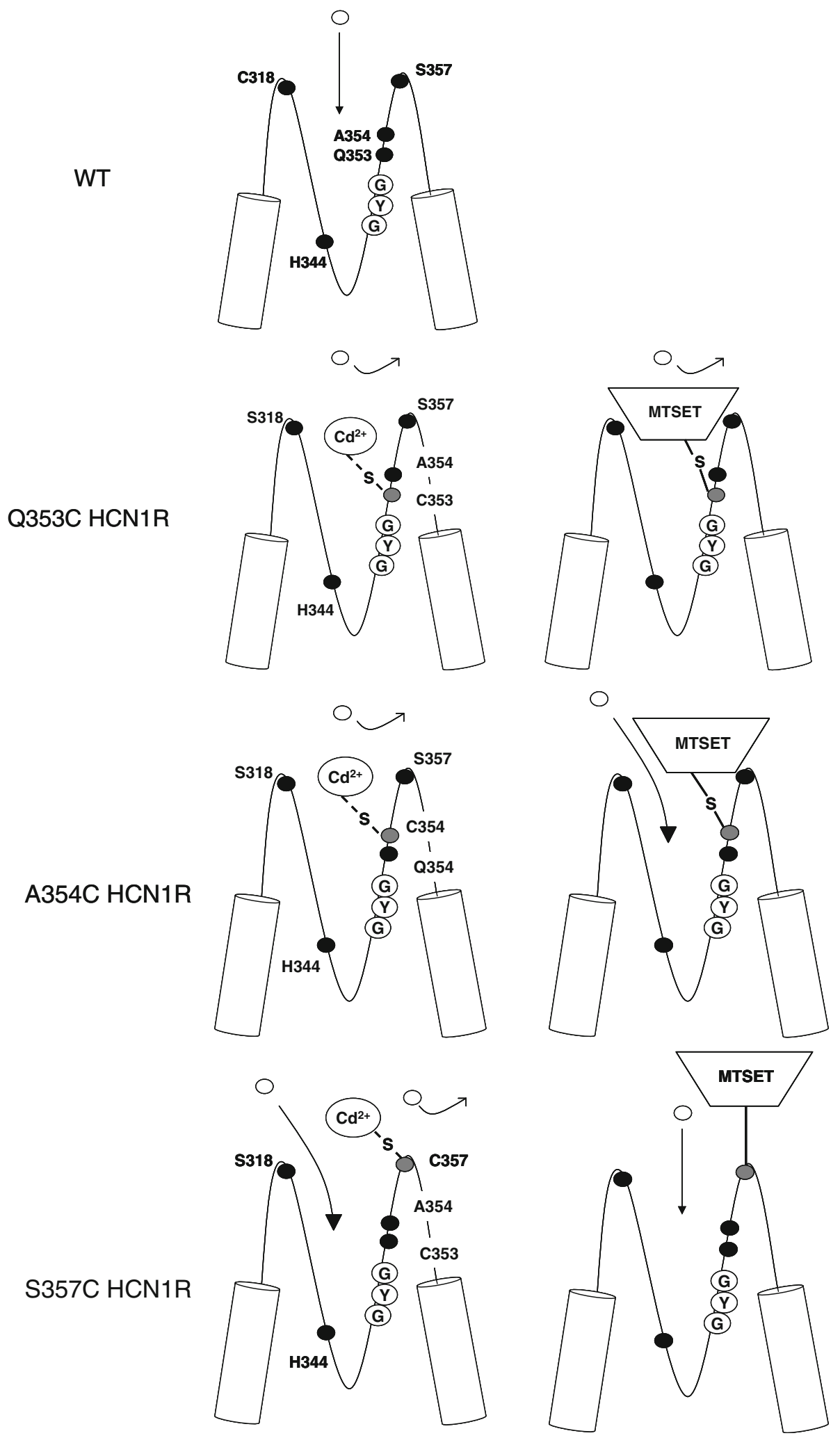

and externally accessible by $\mathrm{Cd}^{2+}$. MTS modification of C318, H344, Q353, and A354, but not S357, alters activation gating, presumably due to their locations in pore regions that undergo dynamic rearrangements. MTS reactivity of $\mathrm{C} 318$, however, is not state dependent as we previously demonstrated (Xue and Li 2002).

Other than the extracellularly accessible residues Q353, A354, and S357, our results also support the notion that 
other P-S6 linker residues studied are also structural and functional determinants of $\mathrm{HCN}$ channels. Although channel proteins were expressed at levels comparable to WT and HCN1-R, HCN functions were completely abolished by substituting any of A352, P355, V356, M358, or S359 with a Cys. Specifically, A352C, Q353C, V356C, S357C, and S359C were capable of cross-linking, although our experiments did not allow us to rule out the possibility of at least one HCN subunit cross-linking with endogenous oocyte proteins. Nonetheless, such resultant dimers could be reduced by DTT coincubation. Whereas the loss-offunction of Q353C, A354C, S357C, and M358C could be rescued after dimer reduction, others remained dysfunctional even after DTT treatment. Although the endogenous C303 in HCN1-R that has not been replaced could crosslink with the inserted sulfhryl of the same subunit to abolish channel functions, this possibility can be ruled out because such an internal disulfide bridge would not lead to the formation of dimers. Assuming that the $\mathrm{HCN}$ pore is analogous to the crystal structure of KcsA (Doyle et al. 1998; Roux and MacKinnon 1999), the predicted distance ( $35 \AA$ ) makes it unlikely for C303 from one monomer to cross-link with an introduced P-S6 cysteine from another monomer. Indeed, disulfide bond formation is a dynamic collisional process that requires at least occasional proximity ( $\sim 3.5 \AA)$ of the free sufhydryls involved with their $\alpha$-carbons oriented at particular angles.

Based on our results, we conclude that the P-S6 linker residues 352-359 are important determinants of the structure-function properties of HCN1 channels. Among these pore residues, Q353, A354, and S357 are exposed to the aqueous phase. The accessibility of Q353 and A354 is state dependent, consistent with an allosteric pore-to-gate coupling model that involves dynamic rearrangements of the extrapore.

Acknowledgments This work was supported by grants from the National Institutes of Health (R01 HL72857 to R.A.L.), Croucher Foundation Fellowship (C.W.S), the University of Hong Kong Seed Funding Programme for Basic Research (HKU 200808159001 to C.W.S), the Hong Kong Research Grant Council General Research Fund (HKU 7747/08 M to C.W.S., H.F.T., and R.A.L.), and the CC Wong Stem Cell Fund (to H.F.T. and R.A.L.).

Open Access This article is distributed under the terms of the Creative Commons Attribution Noncommercial License which permits any noncommercial use, distribution, and reproduction in any medium, provided the original author(s) and source are credited.

\section{References}

Akabas MH, Stauffer DA, Xu M, Karlin A (1992) Acetylcholine receptor channel structure probed in cysteine-substitution mutants. Science 258:307-310
Au KW Siu CW, Lau CP, Tse HF, Li RA (2008) Structural and functional determinants in the S5-P region of $\mathrm{HCN}$-encoded pacemaker channels revealed by cysteine-scanning substitutions. Am J Physiol Cell Physiol 19:136-141

Azene EM, Xue T, Li RA (2003) Molecular basis of the effect of potassium on heterologously expressed pacemaker (HCN) channels. J Physiol 547:349-356

Azene EM, Sang D, Tsang SY, Li RA (2005) Pore-to-gate coupling of HCN channels revealed by a pore variant that contributes to gating but not permeation. Biochem Biophys Res Commun 327:1131-1142

Bell DC, Yao H, Saenger RC, Riley JH, Siegelbaum SA (2004) Changes in local S4 environment provide a voltage-sensing mechanism for mammalian hyperpolarization-activated $\mathrm{HCN}$ channels. J Gen Physiol 123:5-19

Benitah JP, Tomaselli GF, Marban E (1996) Adjacent pore-lining residues within sodium channels identified by paired cysteine mutagenesis. Proc Natl Acad Sci USA 93:7392-7396

Biel M, Schneider A, Wahl C (2002) Cardiac HCN channels: structure, function, and modulation. Trends Cardiovasc Med 12:206-212

Brown HF, Giles W, Noble SJ (1977) Membrane currents underlying activity in frog sinus venosus. J Physiol 271:783-816

Bucossi G, Nizzari M, Torre V (1997) Single-channel properties of ionic channels gated by cyclic nucleotides. Biophys J 72:1165-1181

Chen S, Wang J, Siegelbaum SA (2001) Properties of hyperpolarization-activated pacemaker current defined by coassembly of HCN1 and HCN2 subunits and basal modulation by cyclic nucleotide. J Gen Physiol 117:491-504

Chen S, Wang J, Zhou L, George MS, Siegelbaum SA (2007) Voltage sensor movement and cAMP binding allosterically regulate an inherently voltage-independent closed-open transition in $\mathrm{HCN}$ channels. J Gen Physiol 129:175-188

Creighton TE (1992) Protein folding. W.H. Freeman, New York

DiFrancesco D (1981a) A new interpretation of the pace-maker current in calf Purkinje fibres. J Physiol 314:359-376

DiFrancesco D (1981b) A study of the ionic nature of the pace-maker current in calf Purkinje fibres. J Physiol 314:377-393

DiFrancesco D (1993) Pacemaker mechanisms in cardiac tissue. Annu Rev Physiol 55:455-472

DiFrancesco D (2006) Serious workings of the funny current. Prog Biophys Mol Biol 90:13-25

Doyle DA, Morais Cabral J, Pfuetzner RA, Kuo A, Gulbis JM, Cohen SL, Chait BT, MacKinnon R (1998) The structure of the potassium channel: molecular basis of $\mathrm{K}+$ conduction and selectivity. Science 280:69-77

Flynn GE, Black KD, Islas LD, Sankaran B, Zagotta WN (2007) Structure and rearrangements in the carboxy-terminal region of SpIH channels. Structure 15:671-682

Gauss R, Seifert R, Kaupp UB (1998) Molecular identification of a hyperpolarization-activated channel in sea urchin sperm. Nature 393:583-587

Giorgetti A, Carloni P, Mistrik P, Torre V (2005) A homology model of the pore region of HCN channels. Biophys J 89:932-944

Karlin A, Akabas MH (1998) Substituted-cysteine accessibility method. Methods Enzymol 293:123-145

Kaupp UB, Seifert R (2001) Molecular diversity of pacemaker ion channels. Annu Rev Physiol 63:235-257

Lieu DK, Chan YC, Lau CP, Tse HF, Siu CW, Li RA (2008) Overexpression of $\mathrm{HCN}$-encoded pacemaker current silences bioartificial pacemakers. Heart Rhythm 5:1310-1317

Liu Y, Jurman ME, Yellen G (1996) Dynamic rearrangement of the outer mouth of a K+ channel during gating. Neuron 16:859-867

Ludwig A, Zong X, Jeglitsch M, Hofmann F, Biel M (1998) A family of hyperpolarization-activated mammalian cation channels. Nature 393:587-591 
Prole DL, Yellen G (2006) Reversal of HCN channel voltage dependence via bridging of the S4-S5 linker and Post-S6. J Gen Physiol 128:273-282

Roncaqlia P, Mistrik P, Torre V (2002) Pore topology of the hyperpolarization-activated cyclic nucleotide-gated channel from sea urchin sperm. Biophys J 83:1953-1964

Roux B, MacKinnon R (1999) The cavity and pore helices in the KcsA K+ channel: electrostatic stabilization of monovalent cations. Science 285:100-102

Santoro B, Liu DT, Yao H, Bartsch D, Kandel ER, Siegelbaum SA, Tibbs GR (1998) Identification of a gene encoding a hyperpolarization-activated pacemaker channel of brain. Cell 93:717729
Siu CW, Lieu DK, Li RA (2006) HCN-encoded pacemaker channels: from physiology and biophysics to bioengineering. J Membr Biol 214:115-122

Tsushima RG, Li RA, Backx PH (1997) P-loop flexibility in Na+ channel pores revealed by single- and double-cysteine replacements. J Gen Physiol 110:59-72

Xue T, Li RA (2002) An external determinant in the S5-P linker of the pacemaker $(\mathrm{HCN})$ channel identified by sulfhydryl modification. J Biol Chem 277:46233-46242

Xue T, Marban E, Li RA (2002) Dominant-negative suppression of HCN1- and HCN2-encoded pacemaker currents by an engineered HCN1 construct: insights into structure-function relationships and multimerization. Circ Res 90:1267-1273 